\title{
Cell Signaling In Health And Disease: Think Zinc!
}

\author{
By Guest Editors Stefano L Sensi, Lorella MT Canzoniero and Susan Benoff \\ Online address: http://www.molmed.org \\ doi: 10.2119/2007-00048.Sensi
}

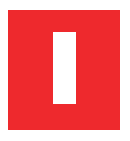

$\mathrm{n}$ this special issue of Molecular Medicine we report on the $6^{\text {th }}$ Zinc Signals conference held last year at the Abbey of Monte Oliveto in Italy. At the meeting, more than 130 researchers from around the globe gathered to discuss the latest advances in the field of $\mathrm{Zn}^{2+}$ biology. Zinc is the second most abundant heavy metal in the human body, essential for proper development and function of brain, skin, reproductive, and digestive systems. Although eukaryotic cells contain as much as $200 \mu \mathrm{M}$ of this ion, the vast majority is tightly bound to intracellular proteins, serving in a functional and/or structural role or catalyzing the activity of more than 300 enzymes and proteins. Participants in the Zinc Signals meeting study the free ionic form (i.e., free or chelatable) $\mathrm{Zn}^{2+}$, whose concentration is considered to be in the picomolar range. This "free- $\mathrm{Zn}^{2+}$ " constitutes a highly dynamic pool that mediates cell signaling.

While, until recently, $\mathrm{Zn}^{2+}$-focused research has been hampered by the lack of adequate tools, technology is catching up and through the availability of transgenic models, $\mathrm{Zn}^{2+}$ specific fluorescent probes, and selective chelators we are now starting to fully reveal the role played by $\mathrm{Zn}^{2+}$ signaling in many physiological and patho-physiological conditions.

A key concept emerging today is that biological systems possess a finely tuned " $\mathrm{Zn}^{2+}$ set-point." Prenatal zinc deficiency impairs brain development and results in serious cognitive deficits later in life. In the reproductive system, $\mathrm{Zn}^{2+}$ has been shown to be essential for testicular development as well as sperm motility. In the colon, the integrity of the epithelial layer is dependent upon zinc, and supplementation is highly effective in prevention of potentially deadly diarrhea. In the pancreatic islets, zinc is required for storage of insulin with which it is co-released, and it has been shown to affect hormone secretion. $\mathrm{Zn}^{2+}$ deficiency is also linked to taste disorders, skin lesions and wound healing. While $\mathrm{Zn}^{2+}$ deficiency can greatly impair function in many biological systems, excessive $\left[\mathrm{Zn}^{2+}\right]$ i can also be deleterious. For instance, $\mathrm{Zn}^{2+}$ has been implicated in $\beta$-amyloid plaque formation in Alzheimer's disease.

At the cellular level, the " $\mathrm{Zn}^{2+}$ set point" is maintained by the concerted activity of $\mathrm{Zn}^{2+}$ many homeostatic systems.

Alteration in the $\mathrm{Zn}^{2+}$ set point can have important consequences. Changes in the $\mathrm{Zn}^{2+}$ concentration, within a "physiological range," have emerged as an important means to modulate signaling. In the central nervous system, for instance, such changes have been shown to regulate the activity of major receptors (GABA, NMDA, and glycine), ion channels, and transporters. Exciting new findings also have started to shed some light on the role played by synaptic $\mathrm{Zn}^{2+}$ in modulating long-term potentiation.

Acute $\mathrm{Zn}^{2+}$ depletion, induced, for example, by cation-specific chelation, can be a potent trigger for apoptosis via inhibition of the caspase system, effects on $\mathrm{Zn}^{2+}$ binding transcription factors, and direct modulation of the ionic exchange between the cytoplasm, the perinuclear compartment, and the nucleoplasm. Intracellular $\mathrm{Zn}^{2+}$ accumulation, on the other hand, mediates toxic effects in a variety of pathological conditions, including cerebral ischemia, brain trauma and epilepsy in addition to $\beta$-cell death during diabetes. Changes in expression of $\mathrm{Zn}^{2+}$ homeostatic proteins, for example, ZIP1 and LIV1, can also be catastrophic, and have been linked to prostate and breast cancer.

Intriguingly, recent evidence demonstrates $\mathrm{Zn}^{2+}$ crosstalk with the other cell signaling molecules, especially the signaling cation "par excellence," $\mathrm{Ca}^{2+}$. Given that the molar potency of $\mathrm{Zn}^{2+}$ in activating/modulating cell signaling pathways is in many cases much higher than $\mathrm{Ca}^{2+}$, it is not far-fetched to envision that $\mathrm{Zn}^{2+}$ will soon compete with $\mathrm{Ca}^{2+}$ for the role of major signaling modulator in cell biology.

The 2006 Zinc Signals conference superbly illustrated the extraordinary variety of aspects related to $\mathrm{Zn}^{2+}$ biology: imaging tools, cell physiology, system physiology, and patho-physiology. Each of these topics is covered in this special issue and represents the cutting edge and new frontiers in a highly dynamic and visible field of biology.

After the outstanding 2006 Zinc Signals meeting, it was clear that the richness of $\mathrm{Zn}^{2+}$-focused research justifies the establishment of an international organization devoted to its study. In January of this year, therefore, we established the Society for Zinc Biology (SZB, www.sfzb.org), and are proud of the overwhelmingly enthusiastic response. By this means, we have successfully assembled the most prominent $\mathrm{Zn}^{2+}$ biologists and are now looking forward to the $1^{\text {st }}$ SZB meeting to be held in February 2008 in Banff, Canada.

Stay tuned for the zinc revolution... 